\title{
A Generalized Two-Dimensional Gaussian Model of Disease Foci of Head Blight of Wheat Caused by Gibberella zeae
}

\author{
T. C. Paulitz, P. Dutilleul, S. H. Yamasaki, W. G. D. Fernando, and W. L. Seaman
}

First and second authors: Department of Plant Science; third author: Department of Natural Resource Sciences, Macdonald Campus of McGill University, Ste. Anne de Bellevue, Quebec H9X 3V9 Canada; and fourth and fifth authors: Agriculture and Agri-Food Canada, Plant Research Centre, Ottawa, Ontario K1A 0C6 Canada.

Current address of W. G. D. Fernando: Department of Plant Science, University of Manitoba, Winnipeg, Manitoba R3T 2N2 Canada. Accepted for publication 1 October 1998.

\begin{abstract}
Paulitz, T. C., Dutilleul, P., Yamasaki, S. H., Fernando, W. G. D., and Seaman, W. L. 1999. A generalized two-dimensional Gaussian model of disease foci of head blight of wheat caused by Gibberella zeae. Phytopathology 89:74-83.

A generalized two-dimensional Gaussian model is proposed to describe disease foci of head blight of wheat in plots $\left(100\right.$ to $\left.2,500 \mathrm{~m}^{2}\right)$ originating from small areas (1 to $\left.16 \mathrm{~m}^{2}\right)$ inoculated with Gibberella zeae-colonized corn kernels. These anisotropic, asymmetrical foci arose from ascospores produced in perithecia. The model is $Z=\exp \left[-\left(A \mathrm{X}^{2}+B \mathrm{Y}^{2}+C \mathrm{XY}+D \mathrm{X}+\right.\right.$ $E \mathrm{Y}+F)]$, in which $Z=$ the incidence of seed or spikelet infection at point $(\mathrm{X}, \mathrm{Y})$ located in the plot, $\exp =$ the exponential function, $\mathrm{X}=$ the abscissa or spatial coordinate of the point along a given axis (approximately parallel to the average wind vector during the period of spore release in these experiments), $\mathrm{Y}=$ the ordinate or spatial coordinate of the point along the axis perpendicular to the $\mathrm{X}$ axis (approximately perpendicular to the wind direction in these experiments), $A$ and $B=$ the quadratic coefficients of the second-order polynomial $A \mathrm{X}^{2}+B \mathrm{Y}^{2}+C \mathrm{XY}+D \mathrm{X}+E \mathrm{Y}+F, C=$ the bilinear coefficient, $D$ and $E=$ the linear coefficients, and $\exp (-F)=$ the incidence of seed or spikelet infection at the focus peak in which $X=0$ and $\mathrm{Y}=0$. The generalized two-dimensional Gaussian model was tested on

axis of symmetry. Its goodness-of-fit $\left(r^{2}\right.$ and adjusted $\left.r^{2}\right)$ was compared with the inverse power, modified inverse power, exponential, and classical Gaussian models. Submodels using only the linear terms, only the quadratic terms, or combinations selected from stepwise regression procedures using various probabilities to enter and to stay and a procedure maximizing the adjusted $r^{2}$ were also considered. Spatial analysis of the residuals was performed using Geary's $c$ coefficient at the first distance class. For the circular and elliptical foci, our model provided a fit similar to the modified inverse power and exponential models. However, for anisotropic foci with one or zero axis of symmetry arising from ascospores influenced by wind direction, the generalized two-dimensional Gaussian model provided a better fit. For these anisotropic foci, the linear term $\mathrm{X}$ but not the quadratic term $\mathrm{X}^{2}$ was generally retained in the model, indicating an exponential gradient in the direction parallel to the wind. In all models, the quadratic term $\mathrm{Y}^{2}$ was retained, along with $\mathrm{Y}$ in some cases, indicating that the gradient in the direction roughly perpendicular to the wind was Gaussian or Gaussian-exponential in shape. The bilinear term $\mathrm{XY}$ provided an indication of the orientation of the focus in relation to the axes of the sampling grid. This model has the versatility and parameters (quadratic, bilinear, and linear) to better describe the anisotropy of foci from wind-dispersed spores.
\end{abstract} data from a circular or isotropic focus, an elliptical or anisotropic focus with two axes of symmetry, and two anisotropic foci with one and zero
Additional keywords: disease gradient.
Head blight of wheat, caused by Gibberella zeae $($ anamorph $=$ Fusarium graminearum), is a monocyclic disease. In eastern Canada, heads of wheat are infected at anthesis by ascospores or macroconidia (19). Macroconidia, which are mostly splash-dispersed, are formed on sporodochia and ascospores are formed in perithecia, both produced on crop debris colonized the previous year. Spores infect the anthers on the wheat heads, and the fungus develops in the young kernels, rachis, and spikelets of the head. Head blight symptoms develop 2 to 3 weeks after infection and include premature senescence of infected spikelets and production of pink sporodochia and mycelium under favorable moist conditions. Because of the limited period of susceptibility of the head, there is probably little secondary infection, except of younger tillers. Although this disease is endemic in eastern Canada, it has generated increasing interest because of devastating epidemics in 1993 and 1994 in the upper midwest of the United States and Manitoba and in the northeastern United States and Ontario in 1996. The epidemiology of the perfect stage of this fungus is poorly understood and has been the focus of collaborative research in Ontario and Quebec since 1992.

Corresponding author: T. C. Paulitz; E-mail address: paulitz@nrs.mcgill.ca

Publication no. P-1998-1123-04R

(C) 1999 The American Phytopathological Society
The gradients of this disease from small area sources of inoculum were studied in inoculated wheat plots in Ontario and Quebec over a 3 -year period (6). By using a strain of G. zeae with a unique colony morphology, we could accurately assess seed infection from samples taken in grid patterns in fields inoculated with colonized corn kernels that produced perithecia and ascospores. Because of the uniform distribution of wheat plants in a field, the high density of plants, and the monocyclic nature of the disease, this provided a model system to study disease foci of an airborne pathogen without the complicating factors of temporal spread. In addition, with a monocyclic disease, the infection and disease gradients will be closely related to the spore dispersal gradients (17) and may provide information about the movement of ascospores. Several workers have recently modeled the spatio-temporal spread of foci. Gottwald (10) used the Gompertz model to describe the temporal progress of citrus scab caused by Elsinoe fawcettii in Florida nurseries and nonlinear regression to describe the three-dimensional response surfaces of isopaths. Ferrandino (7) examined the three-dimensional dispersal of airborne spores by using a traveling wave model to describe the velocity of the isopaths at different distances from the focus. Epidemics of late leaf spot of peanut were described spatially and temporally by looking at the rate of movement of isopaths over time (1). These studies looked at the distribution and spread of foci in naturally infected fields over time, but very few 
studies have attempted to examine in fine detail the shape and gradients of individual foci at one point in time.

Much of the previous work on airborne pathogens has focused on disease gradients measured along line transects in two to four ordinal directions from the foci $(1,18)$. Attempts were made to relate the spread of the foci to wind by comparing the gradients of the line transects in different directions. Lambert et al. (15) attempted to compensate for wind direction in a model based on line transects by inclusion of a sine function based on direction. However, the measurement of disease spread in two dimensions, depicted with contour maps of isopaths, can provide much more descriptive information of disease foci (6) and can also provide the basis for a two-dimensional (2D) spatial model.

Three basic empirical models have been used to describe dispersal gradients (16). The inverse power law model (model 1) proposed by Gregory (12) assumes that the amount of inoculum, disease, or infection $(y)$ is inversely proportional to the power of the distance from the source of inoculum $(x)$. The equation is

$$
y=a x^{-b}
$$

in which $a$ is the amount of disease at 1 unit of distance from the source and $b$ is a coefficient for the slope or gradient. However, a major drawback of this model is that $y$ approaches infinity as $x=$ 0 ; therefore, this model will overestimate the disease at the source. Mundt (18) modified this model with a truncation factor $c$ (model 2) to allow for a finite number of infections at the source. His model is

$$
y=a^{\prime}\left(x^{\prime}+c\right)^{-b^{\prime}}
$$

in which $x^{\prime}$ is the distance from the center of the inoculum source to the center of the point of disease measurement, $a^{\prime}$ is the infection level at $1-c$ units of distance from the source, and $b^{\prime}$ is a coefficient for the slope or rate of decrease in disease with distance from the inoculum source.

The simple negative exponential model (model 3) of Kiyosawa and Shiyomi (14) assumes the disease decreases exponentially with distance. The equation is

$$
y=a \cdot \exp (-d x)
$$

in which $a$ is the amount of disease at the source and $d$ is the coefficient for the slope or gradient. This model has the advantage of better estimating disease at the source or center of the focus than would the power model, but still often underestimates it $(15,16)$. Also, since the slope $-d$ in a linearized equation has the dimension of length ${ }^{-1}$, the half-distance can be calculated as $0.693 / d$, which gives another way of looking at the gradient. In equations 1 and $2,-b$ and $-b^{\prime}$ also represent the actual slope in a linearized equation.

The model (model 4) proposed by Kampmeijer and Zadoks (13) assumes a normal or Gaussian distribution of disease around a point source. Their model is

$$
y=a /\left(2 \pi s^{2}\right) \cdot \exp \left[-x^{2} /\left(2 s^{2}\right)\right]
$$

in which $y=$ amount of disease at $x$ distance from the inoculum source, $a /\left(2 \pi s^{2}\right)=$ the amount of disease at the source, and $s^{2}$ is a coefficient for the variance of $y$.

Both models 1 and 3 have fixed slopes and shapes, which may give a poorer fit of the data away from the source. To overcome this limitation, Lambert et al. (15) proposed a more general model

$$
a \cdot \exp \left(-x^{n}\right)
$$

which incorporates aspects of the inverse power (equation 1), negative exponential (equation 3), and Gaussian (equation 4) models.

However, these models based on one-dimensional (1D) gradients have not and cannot be used for 2D data of disease foci, except through unidirectional cross-sections or after circular averaging at given distances from the center of the inoculum source. The objec- tive of this paper was to construct a flexible, versatile 2D model for disease foci, incorporating models 3 and 4. Disease gradients of airborne pathogens are often steeper on the upwind than the downwind side of foci and are often different between directions parallel or perpendicular to the wind direction. Therefore, our model will also take into account both isotropic and anisotropic shapes of foci from airborne pathogens influenced by wind. The former are characterized by an infinite number of axes of symmetry and the latter by, at most, two axes of symmetry. Data from disease foci of G. zeae in inoculated wheat plots (6) were used to illustrate and test our model and compare it with previously described models.

\section{MATERIALS AND METHODS}

Model and submodels. Since previous work (6) had shown that disease foci from ascospore inoculum were anisotropic (i.e., dispersion not equal in all directions) and might be asymmetrical, usually in the direction parallel to the average wind vector during the period of spore release, a model was constructed to describe this asymmetry along the $\mathrm{X}$ versus $\mathrm{Y}$ axis and the difference in the slope of the gradient between the upwind and downwind side of the plots. Our model is

$$
Z=\exp \left[-\left(A \mathrm{X}^{2}+B \mathrm{Y}^{2}+C \mathrm{XY}+D \mathrm{X}+E \mathrm{Y}+F\right)\right]
$$

in which $Z=$ the incidence of seed or spikelet infection at point $(\mathrm{X}, \mathrm{Y})$ located in the plot, $\exp =$ the exponential function, $\mathrm{X}=$ the abscissa or spatial coordinate of the point along a given axis (approximately parallel to the average wind vector during the period of spore release in our experiments), $\mathrm{Y}=$ the ordinate or spatial coordinate of the point along the axis perpendicular to the $\mathrm{X}$ axis (i.e., approximately perpendicular to the wind direction), $A$ and $B=$ the quadratic coefficients of the second-order polynomial $A X^{2}+$ $B Y^{2}+C X Y+D X+E Y+F, C=$ the bilinear coefficient, $D$ and $E=$ the linear coefficients, and $\exp (-F)=$ the incidence of seed or spikelet infection at the focus peak in which $\mathrm{X}=0$ and $\mathrm{Y}=0$. Since the maximum seed infection (ranging from 70 to $85 \%$ ) was much higher than the proportion of spikelets diseased, the proportion of seed infection was transformed with the multiple infection correction $\ln [1 /(1-y)](11)$ to account for multiple infections due to the high levels of inoculum generated with our inoculation procedure. Figure 1 shows an idealized anisotropic focus with one axis of symmetry (X horizontal axis) in the Cartesian system centered on the focus peak formed by axes $\mathrm{X}$ and $\mathrm{Y}$ and with grid nodes (dots) to represent the sampled data points.

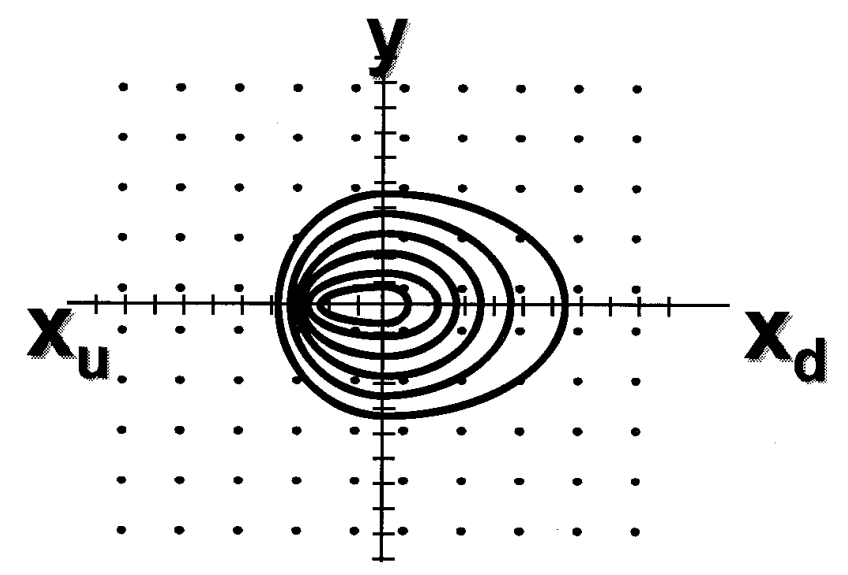

Fig 1. Idealized anisotropic focus with one axis of symmetry (i.e., the horizontal axis $\mathrm{X}$ ) in Cartesian coordinates with grid nodes (sampled data points) represented by dots. $X_{u}=$ direction parallel to wind vector on the upwind side of the focus; $X_{d}=$ direction parallel to wind vector on the downwind side of the focus. 
In such a case, our model was fitted in two parts, upwind and downwind of the $\mathrm{Y}$ axis. For points on the $\mathrm{Y}$ axis itself $(\mathrm{X}=0)$, the two fitted values from each fitted model (upwind and downwind) were averaged together.

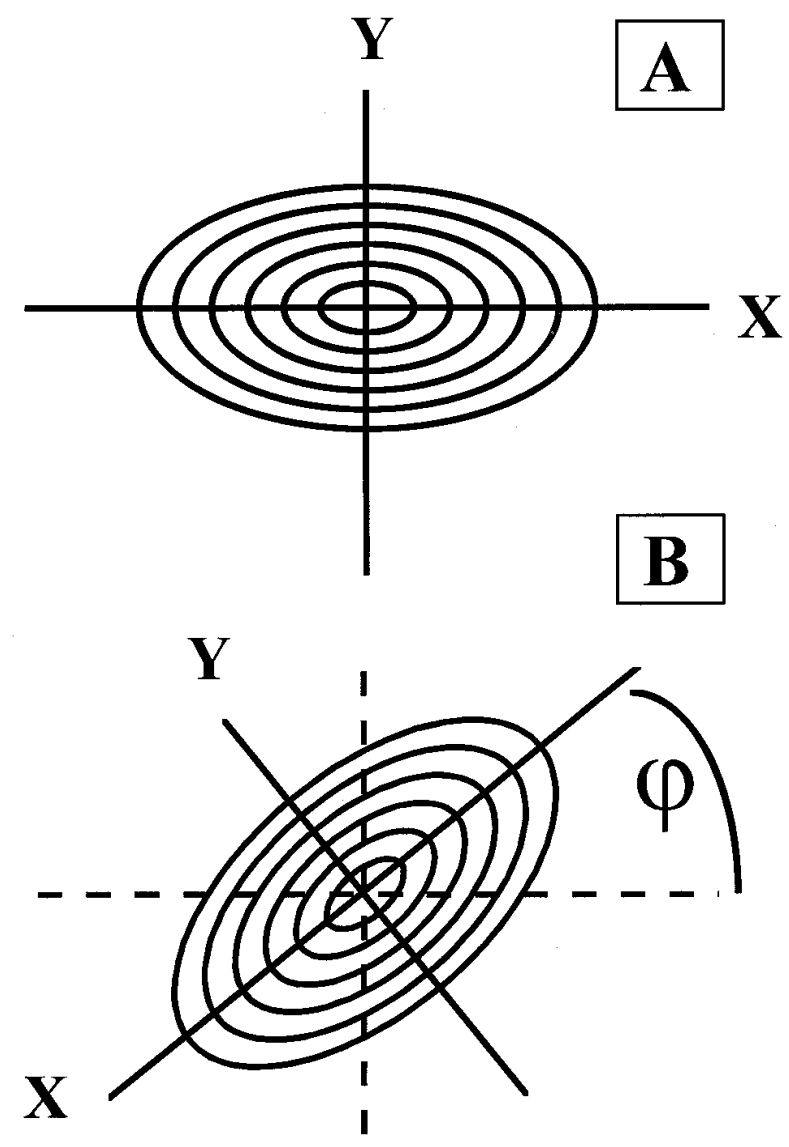

Fig. 2. Idealized elliptical (anisotropic with two axes of symmetry) focus. A, Focus represented by the following version of equation $6: Z=\exp \left[-\left(A X^{2}+\right.\right.$ $\left.B \mathrm{Y}^{2}+F\right)$ ], in which $A>0, B>0, A<B$, and $C=0$. In this version, the major and minor axes of the ellipse are aligned with the axes of the sampling grid. B, Focus in which major and minor axes are not aligned with the axes of the sampling grid. The focus is represented by the following version of equation 6 : $Z=\exp \left[-\left(A \mathrm{X}^{2}+B \mathrm{Y}^{2}+C \mathrm{XY}+F\right)\right]$, in which $A>0, B>0, A<B$, and $C<0$. The angle $\varphi=1 / 2 \tan ^{-1}[C /(A-B)]$.

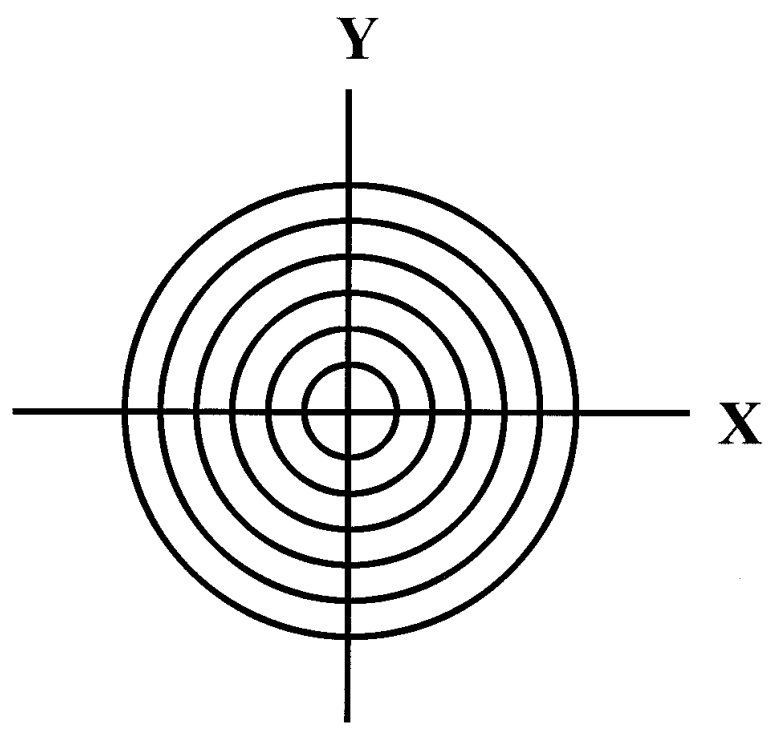

Fig. 3. Idealized circular (isotropic) focus.
The 1D gradient along Y (i.e., perpendicular to the wind), following a cross-section of $\mathrm{X}$ (i.e., for a given $\mathrm{X}$ abscissa), is given by the equation

$$
Z=\exp \left[-\left(B \mathrm{Y}^{2}+G \mathrm{Y}+H\right)\right]
$$

in which $G=C \mathrm{X}+E$ and $H=A \mathrm{X}^{2}+D \mathrm{X}+F$.

With $\mathrm{X}_{\mathrm{u}}$ and $\mathrm{X}_{\mathrm{d}}=$ the abscissa upwind and downwind from the focus peak, respectively, the $1 \mathrm{D}$ gradient along $X_{u}$ following a crosssection of $\mathrm{Y}$ (i.e., for a given $\mathrm{Y}$ ordinate) is represented by the equation

$$
Z=\exp \left[-\left(A \mathrm{X}_{\mathrm{u}}^{2}+G^{\prime} \mathrm{X}_{\mathrm{u}}+H^{\prime}\right)\right]
$$
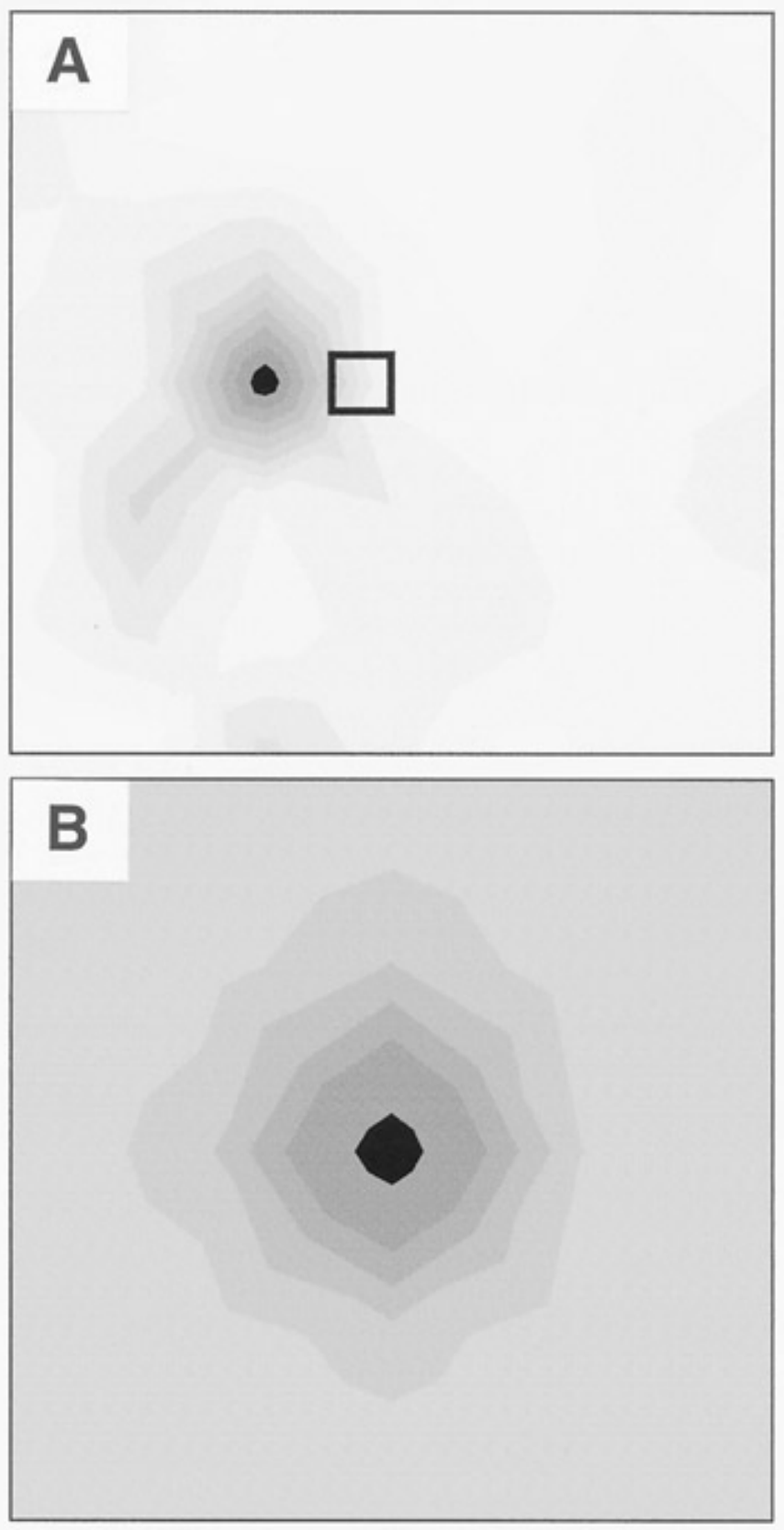

Fig. 4. Contour maps of incidence of seed infection derived from A, observed data and B, model-fitted data of circular focus, Ottawa, 1994. A, Maximum isopath $=66 \%$, minimum $=0 \%$, and contour interval $=6 \%$. $\mathbf{B}$, Maximum isopath $=1.055(65 \%)$, minimum $=0 \%$, and contour interval $=0.15(16.3 \%)$. The generalized Gaussian full model was used for fitting. Raw data were transformed with the multiple infection correction $\ln [1 /(1-y)]$, in which $y=$ incidence of seed infection. Values in parentheses are back-transformed values. The plot was $25 \times 25 \mathrm{~m}$, and the inoculated area (black square) was $1 \times 1 \mathrm{~m}$. Arrows represent north and average nightly wind vector during 1 to 31 July. 
in which $G^{\prime}=C \mathrm{Y}+D$ and $H^{\prime}=B \mathrm{Y}^{2}+E \mathrm{Y}+F$. The estimates of parameters $A, B, C, D, E$, and $F$ in equation 8 come from the fitting of equation 6 to the upwind part of the data. The $1 \mathrm{D}$ gradient along $\mathrm{X}_{\mathrm{d}}$ following a cross-section of $\mathrm{Y}$ is represented by the equation

$$
Z=\exp \left[-\left(A \mathrm{X}_{\mathrm{d}}^{2}+G^{\prime \prime} \mathrm{X}_{\mathrm{d}}+H^{\prime \prime}\right)\right]
$$

in which $G^{\prime \prime}=C \mathrm{Y}+D$ and $H^{\prime \prime}=B \mathrm{Y}^{2}+E \mathrm{Y}+F$. The estimates of parameters $A, B, C, D, E$, and $F$ in equation 9 come from the fitting of equation 6 to the downwind part of the data.

This model can also be applied to the anisotropic case with elliptical shape (Fig. 2). This focus is symmetrical along a major and minor axis (Fig. 2A, X and Y, and Fig. 2B, X and Y shifted by an angle $\varphi$ ). In such a case, parameters $A$ and $B$ in equation 6 are restricted to be positive, and parameters $D$ and $E$ are 0 , which leads to the equation

$$
Z=\exp \left[-\left(A \mathrm{X}^{2}+B \mathrm{Y}^{2}+C \mathrm{XY}+F\right)\right]
$$

in which $C=0$ if the major and minor axes coincide with the $\mathrm{X}$ and Y axes (Fig. 2A), and $C \neq 0$ otherwise (Fig. 2B). The 1D gradient along Y follows from equation 7 with $A>0, B>0, D=0$, and $E=$ 0 . The 1D gradient along $X$ follows from an equation similar to equation 8 , in which $\mathrm{X}_{\mathrm{u}}=\mathrm{X}, A>0, B>0, D=0, E=0$, and the estimates of parameters $A, B, C$, and $F$ come from the fitting of equation 10 to the complete data set.

In the simplest example, the gradient would be the same in all directions (i.e., isotropic), with a circular or radially symmetric cross-sectional shape of the focus (Fig. 3). Parameters $A$ and $B$ in equation 6 are then restricted to be equal and positive, all other parameters (except $F$ ) are 0 , and gradients from the peak of the focus would be represented by the equation

$$
Z=\exp \left[-A\left(\mathrm{X}^{2}+\mathrm{Y}^{2}\right)-F\right]
$$

which is equivalent to the classical 1D Gaussian model (equation 4), since $\mathrm{X}^{2}+\mathrm{Y}^{2}$ represents the squared Euclidean distance (represented by $x^{2}$ in equation 4 ) of point $(\mathrm{X}, \mathrm{Y})$ from the peak of focus, $A$ performs the function of $1 /\left(2 s^{2}\right)$, and $\exp (-F)$ performs the function of $a /\left(2 \pi s^{2}\right)$. Equation 11 can also be seen as a particular case of equation 10 in which $A$ and $B$ are restricted to be equal and $C$ is 0 .

Furthermore, it must be noted that the generalized 2D Gaussian model (equation 6) also includes the classical exponential model (equation 3 ) and its $2 \mathrm{D}$ form as particular cases or submodels. In fact, setting $A=0, B=0$, and $C=0$ in equation 6 results in an anisotropic (i.e., the rate of decrease in disease with distance may differ between the $\mathrm{X}$ and $\mathrm{Y}$ directions) exponential model. Imposing to that the condition $D=E$ leads to equation 3. Other submodels of the generalized 2D Gaussian model were already mentioned above; these were the anisotropic case with elliptical shape and the circular or radially symmetric cross-sectional shape of the focus. In its most general form, equation 6 may provide a mixture of both

TABLE 1. Model performance with case 1, a circular (isotropic) focus, Ottawa, 1994

\begin{tabular}{lcccc}
\hline $\begin{array}{l}\text { Sample size/ } \\
\text { goodnes-of-fit } \\
\text { statistic }\end{array}$ & $\begin{array}{c}\text { Unmodified } \\
\text { inverse power }\end{array}$ & $\begin{array}{c}\text { Modified } \\
\text { inverse power }\end{array}$ & $\begin{array}{l}\text { Exponential } \\
\text { n=36 }\end{array}$ & $\begin{array}{c}\text { Classical } \\
\text { Gaussian }\end{array}$ \\
\hline$r^{2}$ & 0.13 & 0.29 & 0.15 & \\
$\quad$ Adjusted $r^{2}$ & 0.10 & 0.27 & 0.13 & 0.08 \\
$\quad P$ & 0.04 & $<0.01$ & 0.01 & 0.06 \\
$n=25$ & & & & \\
$\quad r^{2}$ & 0.11 & 0.33 & 0.24 & 0.13 \\
$\quad$ Adjusted $r^{2}$ & 0.06 & 0.30 & 0.20 & 0.09 \\
$P$ & 0.14 & $<0.01$ & 0.02 & 0.09 \\
$n=9$ & & & & \\
$\quad r^{2}$ & 0.01 & 0.58 & 0.45 & 0.26 \\
Adjusted $r^{2}$ & -0.18 & 0.52 & 0.36 & 0.13 \\
$P$ & 0.79 & 0.03 & 0.07 & 0.20 \\
\hline
\end{tabular}

types of cross-sections. However, our model does not incorporate the power or modified power model.

Experimental data. Data for all model verifications were provided by experiments described in Fernando et al. (6). The seed or spikelet infection data were taken from grid nodes sampled in wheat plots inoculated in the center with corn kernels colonized with $G$. zeae or sprayed with macroconidia of $F$. graminearum.

Case 1: circular (isotropic) focus. Data were provided from a 1994 experiment in Ottawa, Ontario (figure 5D in literature citation 6). The plot size was $25 \times 25 \mathrm{~m}$ with a $7 \times 7$ sampling grid. Inoculum was applied to the center $1 \mathrm{~m}^{2}$. No additional misting or irrigation was used.

Case 2: elliptical (anisotropic with two axes of symmetry) focus. Data were provided from a 1993 experiment in Ottawa, Ontario (figure $3 \mathrm{C}$ in literature citation 6). The plot size was $10 \times 10 \mathrm{~m}$ with a $6 \times 6$ sampling grid. Macroconidia $\left(5 \times 10^{4}\right.$ spores per $\left.\mathrm{ml}\right)$ were sprayed on the center $1 \mathrm{~m}^{2}$ of heads twice during anthesis. The plot was misted in the day during the anthesis period.

TABLE 2. Generalized two-dimensional Gaussian full model and submodels for case 1 , circular (isotropic) focus

\begin{tabular}{lccccc}
\hline Model/selection & $n$ & $\begin{array}{c}\text { Number of } \\
\text { parameters }^{\mathrm{a}}\end{array}$ & $r^{2}$ & Adjusted $r^{2}$ & $\begin{array}{c}P \text { value } \\
\text { for model }\end{array}$ \\
\hline Full & 33 & 6 & 0.32 & 0.19 & 0.05 \\
No X or Y & 33 & 4 & 0.29 & 0.21 & 0.02 \\
X and Y & 33 & 3 & $<0.001$ & -0.07 & 0.99 \\
Stepwise 0.15 & 33 & 3 & 0.24 & 0.19 & 0.02 \\
Stepwise 0.20 & 33 & 4 & 0.29 & 0.21 & 0.02 \\
Stepwise 0.25 & 33 & 5 & 0.32 & 0.23 & 0.02 \\
Max. adjusted $r^{2}$ & 33 & 5 & 0.32 & 0.23 & 0.02 \\
Full & 23 & 6 & 0.53 & 0.40 & 0.01 \\
No X or Y & 23 & 4 & 0.25 & 0.13 & 0.13 \\
X and Y & 23 & 3 & 0.15 & 0.06 & 0.20 \\
Stepwise 0.15 & 23 & 6 & 0.53 & 0.40 & 0.01 \\
Stepwise 0.20 & 23 & 6 & 0.53 & 0.40 & 0.01 \\
Stepwise 0.25 & 23 & 6 & 0.53 & 0.40 & 0.01 \\
Max. adjusted $r^{2}$ & 23 & 6 & 0.53 & 0.40 & 0.01 \\
Full & 9 & 6 & 0.43 & -1.01 & 0.88 \\
No X or Y & 9 & 4 & 0.29 & -0.23 & 0.68 \\
X and Y & 9 & 3 & 0.19 & -0.13 & 0.58 \\
Stepwise 0.15 & 9 & No model & & & \\
Stepwise 0.20 & 9 & No model & & & \\
Stepwise 0.25 & 9 & 2 & 0.22 & 0.09 & 0.24 \\
Max. adjusted $r^{2}$ & 9 & 3 & 0.37 & 0.11 & 0.32 \\
\hline ancludes & & & & &
\end{tabular}

a Includes intercept.

${ }^{\mathrm{b}}$ For stepwise regressions, the number denotes the probability level at which regressors enter the model.

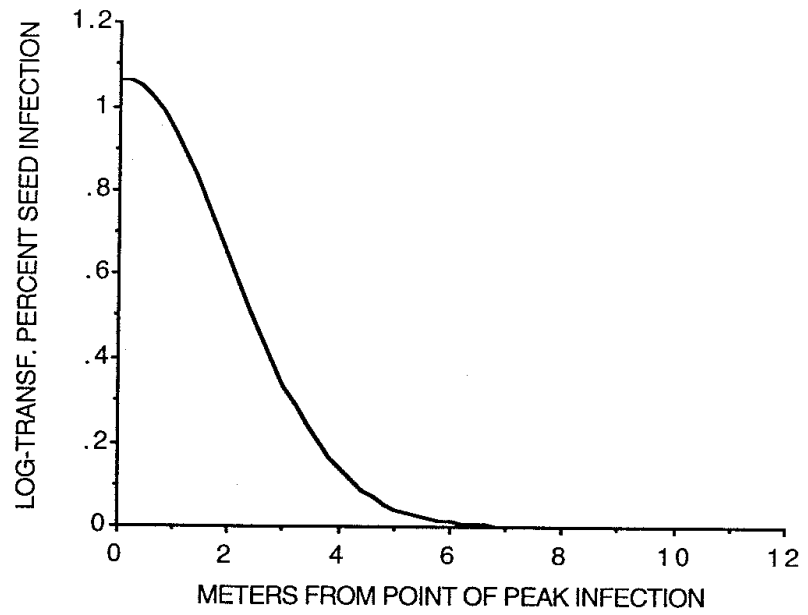

Fig. 5. One-dimensional gradient of seed infection derived from the generalized two-dimensional Gaussian submodel defined by equation 11 for the circular focus in Figure 4. 
Case 3: anisotropic focus with one axis of symmetry. Data was taken from a 1994 experiment in Ottawa, Ontario (figure 5C in literature citation 6 ). The plot size was $25 \times 25 \mathrm{~m}$ with a $7 \times 7$ sampling grid. Corn kernel inoculum was applied to the center $1 \mathrm{~m}^{2}$. No additional irrigation or misting was used.

Case 4: anisotropic focus with zero axis of symmetry. This example was from a 1993 experiment at Ste. Anne de Bellevue, Quebec, in a $10 \times 10-\mathrm{m}$ plot with inoculated corn kernels applied to the center $4 \times 4-\mathrm{m}$ section (figure $1 \mathrm{~B}$ in literature citation 6 ). Samples were taken on the nodes of a $5 \times 5$ grid, and spikelet
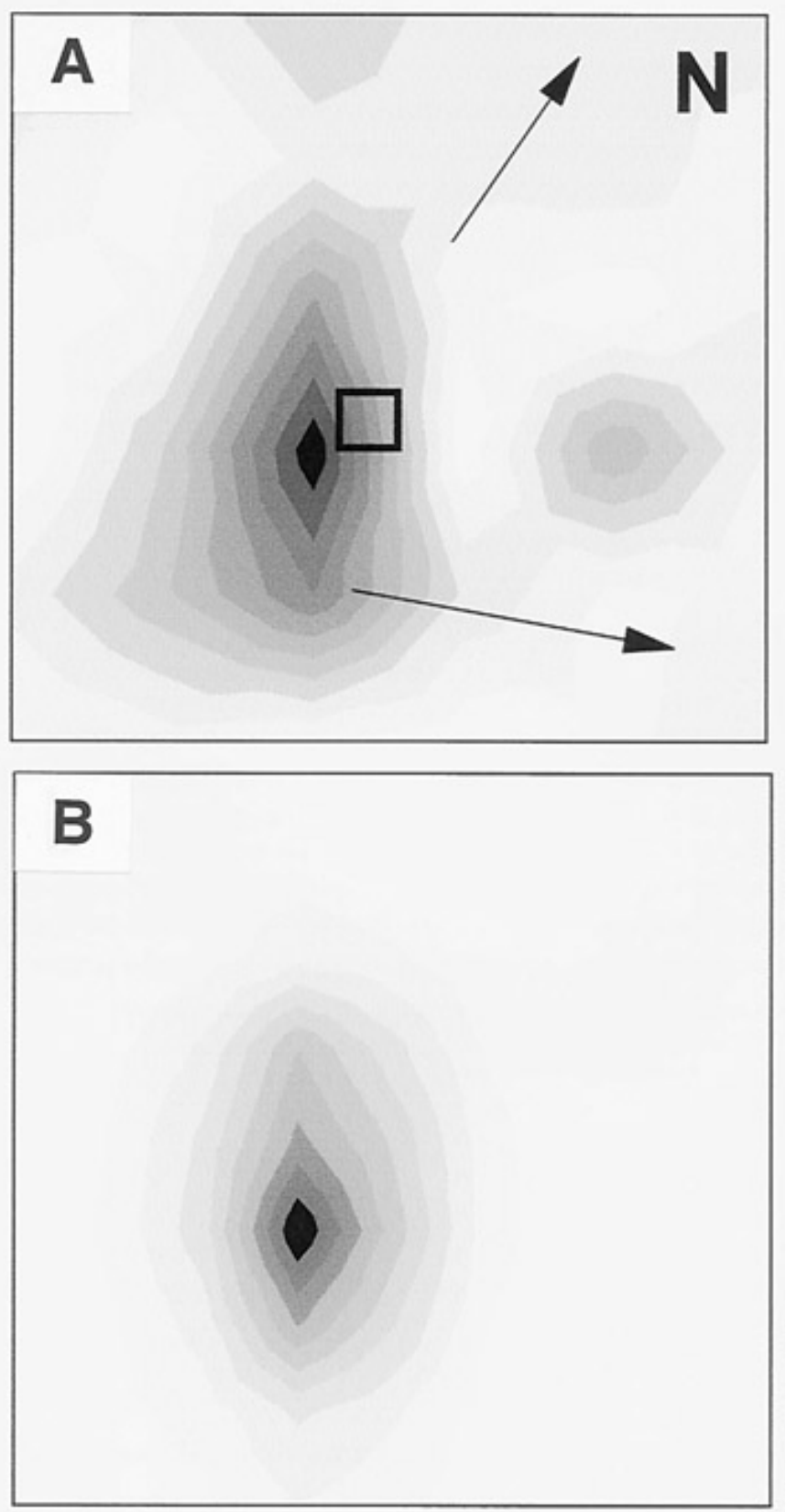

Fig. 6. Contour maps of incidence of seed infection derived from A, observed data and $\mathbf{B}$, model-fitted data of elliptical (anisotropic with two axes of symmetry) focus, Ottawa, 1993. A, Maximum isopath $=54 \%$, minimum $=0 \%$, and contour interval $=5 \%$. $\mathbf{B}$, Maximum isopath $=0.70$ $(50.3 \%)$, minimum $=0 \%$, and contour interval $=0.07(7 \%)$. The generalized two-dimensional Gaussian full model was used for fitting. Raw data were transformed with the multiple infection correction $\ln [1 /(1-y)]$, in which $y=$ incidence of seed infection. Values in parentheses are backtransformed values. The plot was $10 \times 10 \mathrm{~m}$, and the inoculated area was $1 \times 1 \mathrm{~m}$. Plots were misted during anthesis. Arrows represent north and average nightly wind vector during 1 to 22 July. infection was measured 3 weeks after anthesis. No additional misting or irrigation was used.

Model testing and verification. The unmodified inverse power law model (equation 1), the inverse power law model modified by Mundt (equation 2), the negative exponential model of Kiyosawa and Shiyomi (equation 3), the classical Gaussian model of Kampmeijer and Zadoks (equation 4), the generalized 2D Gaussian model in the full version (equation 6), and two submodels of it (one with $D=$ 0 and $E=0$ [no X and Y terms] and the other with $A=0, B=0$, and $C=0$ [no $\mathrm{X}^{2}, \mathrm{Y}^{2}$, and $\mathrm{XY}$ terms]) were each tested for goodness-of-fit to the observed data with the coefficient of determination $\left(r^{2}\right)$. This was carried out after $\ln$ transformation (for the modified inverse power model, after adjustment of $x^{\prime}$ to the $c$ estimated in a preliminary nonlinear step), using procedure REG of SAS version 6 (SAS Institute Inc., Cary, NC). The nonlinear step for the modified inverse power model was carried out by SAS procedure NLIN. A stepwise regression procedure (3) was also performed to select possible submodels of the generalized 2D Gaussian model other than the two initially considered. This was carried out with option STEPWISE of SAS procedure REG and probabilities to enter and to stay of 0.15 (default value), 0.20 , and 0.25 . The same procedure REG provides, with option ADJRSQ, the model maximizing the adjusted $r^{2}$ (described below).

TABLE 3. Model performance with case 2, an elliptical (anisotropic with two axes of symmetry) focus, Ottawa, 1993

\begin{tabular}{|c|c|c|c|c|}
\hline $\begin{array}{l}\text { Sample size/ } \\
\text { goodnes-of- } \\
\text { fit statistic }\end{array}$ & $\begin{array}{l}\text { Unmodified } \\
\text { inverse power }\end{array}$ & $\begin{array}{c}\text { Modified } \\
\text { inverse power }\end{array}$ & Exponential & $\begin{array}{l}\text { Classical } \\
\text { Gaussian }\end{array}$ \\
\hline \multicolumn{5}{|l|}{$n=36$} \\
\hline$r^{2}$ & 0.02 & 0.16 & 0.04 & 0.01 \\
\hline Adjusted $r^{2}$ & -0.01 & 0.13 & 0.02 & -0.02 \\
\hline$P$ & 0.39 & 0.02 & 0.21 & 0.61 \\
\hline \multicolumn{5}{|l|}{$n=25$} \\
\hline$r^{2}$ & 0.16 & 0.23 & 0.25 & 0.17 \\
\hline Adjusted $r^{2}$ & 0.12 & 0.20 & 0.22 & 0.14 \\
\hline$P$ & 0.05 & 0.02 & 0.01 & 0.04 \\
\hline \multicolumn{5}{|l|}{$n=9$} \\
\hline$r^{2}$ & 0.05 & 0.24 & 0.26 & 0.23 \\
\hline Adjusted $r^{2}$ & -0.11 & 0.13 & 0.16 & 0.11 \\
\hline$P$ & 0.60 & 0.18 & 0.16 & 0.20 \\
\hline
\end{tabular}

TABLE 4. Generalized two-dimensional Gaussian full model and submodels for case 2, an elliptical (anisotropic with two axes of symmetry) focus

\begin{tabular}{lccccc}
\hline Model/selection & $n$ & $\begin{array}{c}\text { Number of } \\
\text { parameters }^{\text {a }}\end{array}$ & $r^{2}$ & Adjusted $r^{2}$ & $\begin{array}{c}P \text { value } \\
\text { for model }\end{array}$ \\
\hline Full & 36 & 6 & 0.10 & -0.05 & 0.66 \\
No X or Y & 36 & 4 & 0.06 & -0.02 & 0.56 \\
X and Y & 36 & 3 & 0.02 & -0.03 & 0.66 \\
Stepwise 0.15 & 36 & No model & & & \\
Stepwise 0.20 & 36 & No model & & & \\
Stepwise 0.25 & 36 & No model & & & \\
Max. adjusted $r^{2}$ & 36 & 3 & 0.07 & 0.01 & 0.31 \\
Full & 25 & 6 & 0.23 & 0.03 & 0.36 \\
No X or Y & 25 & 4 & 0.23 & 0.12 & 0.14 \\
X and Y & 25 & 3 & 0.01 & -0.08 & 0.92 \\
Stepwise 0.15 & 25 & 2 & 0.15 & 0.12 & 0.05 \\
Stepwise 0.20 & 25 & 2 & 0.15 & 0.12 & 0.05 \\
Stepwise 0.25 & 25 & 2 & 0.15 & 0.12 & 0.05 \\
Max. adjusted $r^{2}$ & 25 & 3 & 0.20 & 0.12 & 0.10 \\
Full & 9 & 6 & 0.80 & 0.46 & 0.25 \\
No X or Y & 9 & 4 & 0.57 & 0.32 & 0.20 \\
X and Y & 9 & 3 & 0.22 & -0.03 & 0.46 \\
Stepwise 0.15 & 9 & 2 & 0.56 & 0.49 & 0.02 \\
Stepwise 0.20 & 9 & 2 & 0.56 & 0.49 & 0.02 \\
Stepwise 0.25 & 9 & 4 & 0.78 & 0.65 & 0.04 \\
Max. adjusted $r^{2}$ & 9 & 4 & 0.78 & 0.65 & 0.04 \\
\hline
\end{tabular}

${ }^{a}$ Includes intercept.

${ }^{\mathrm{b}}$ For stepwise regressions, the number denotes the probability level at which regressors enter the model. 
All the models were compared with each other using an adjusted $r^{2}$ calculated from $r^{2}$ as follows

$$
\text { adjusted } r^{2}=1-n\left(1-r^{2}\right) /(n-p)
$$

in which $n=$ the number of observations used in fitting the model and $p=$ the number of parameters of the model. This adjusts each model for the number of parameters and provides a better comparison.

A probability of significance was calculated for each model, using the ANOVA $F$-ratio test performed on the linearized (i.e., after $\ln$ transformation) model. When possible, the performance of the models was also tested on a scaled-down area (e.g., $6 \times 6,5 \times 5,3 \times 3$ nodes), by finally using the grid samples from just the disease focus.

Model verification was completed by the spatial analysis of residuals of the model maximizing the adjusted $r^{2}$ among the classical models defined by equations 1 to 4 versus the model maximizing the adjusted $r^{2}$ among the full version and various submodels of the generalized 2D Gaussian model. This spatial analysis was based on Geary's $c$ coefficient $(2,5,9)$ and was performed using procedure AUTOCOR of software "R" (P. Legendre and A. Vaudor, Département de sciences biologiques, Université de Montréal). Following the procedure of Durbin and Watson (4) while extending it to the 2D spatial case, we restricted the analysis of Geary's $c$ to the first distance class corresponding to the nearest-neighbor data points.
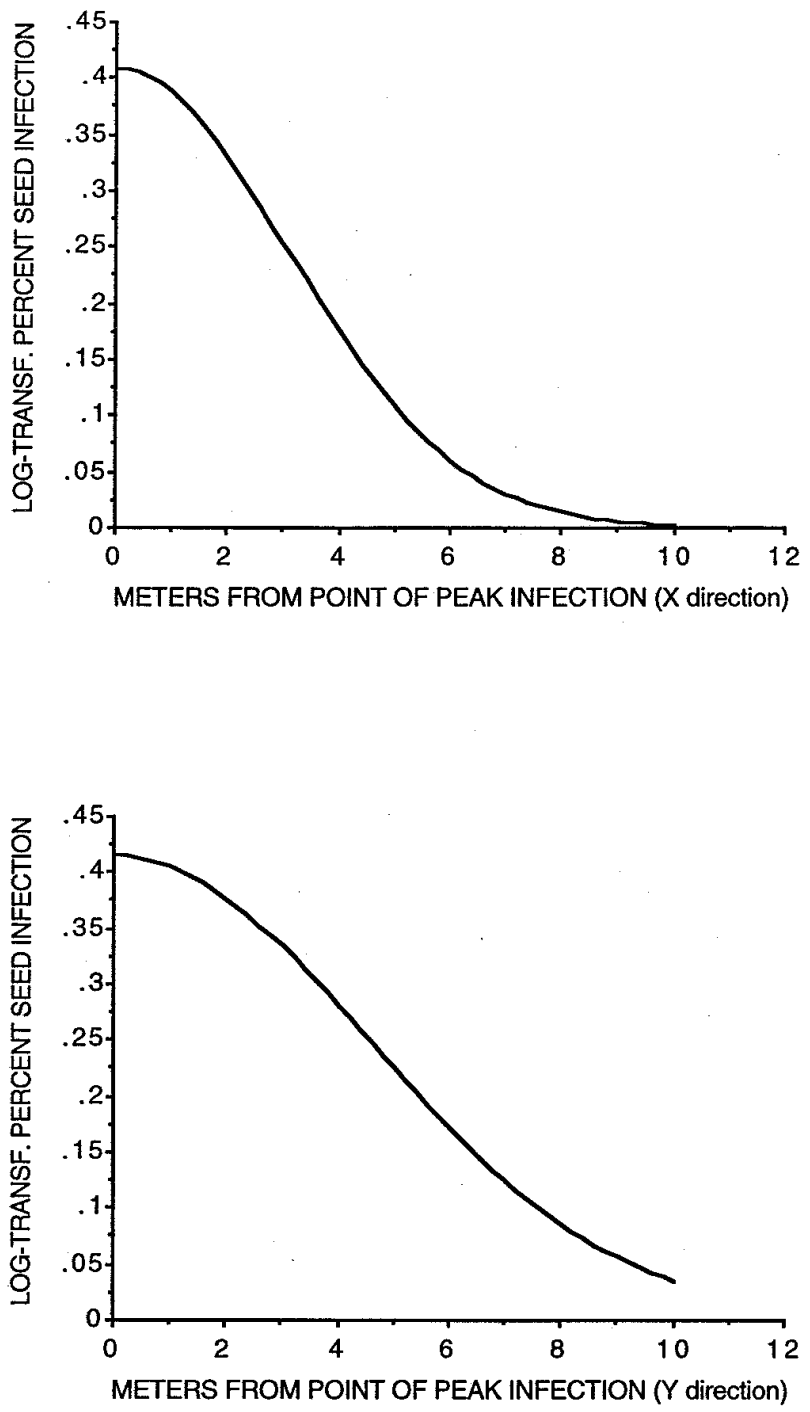

Fig. 7. One-dimensional gradients of seed infection derived from the generalized Gaussian submodel defined by equation 10 for the elliptical focus in Figure 6.

\section{RESULTS}

Case 1: circular. The observed data and the data fitted using the generalized 2D Gaussian model are mapped in Figure 4A and B, respectively. The goodness-of-fit of the other models are presented in Table 1, and results for the generalized 2D Gaussian model and its submodels are presented in Table 2 . When data from the $6 \times 6$ grid were used, which included points away from the focus, the modified inverse power model provided the best fit, followed by the
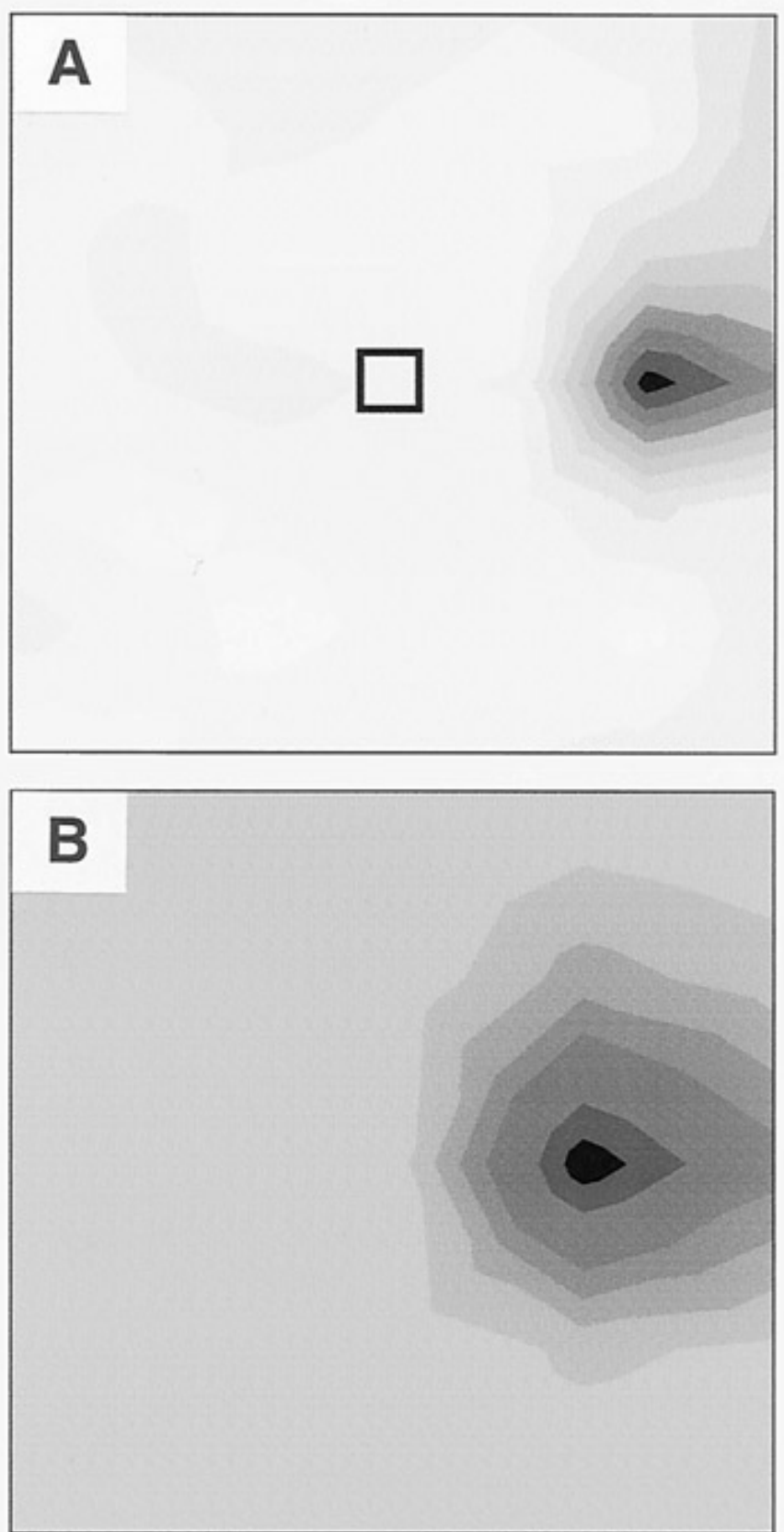

Fig. 8. Contour maps of incidence of seed infection derived from A, observed data and $\mathbf{B}$, model-fitted data of an anisotropic focus with one axis of symmetry (parallel to the $\mathrm{X}$ axis), Ottawa, 1994. A, Maximum isopath $=84 \%$, minimum $=2 \%$, and contour interval $=8 \%$. $\mathbf{B}$, Maximum isopath $=1.93$ $(85.5 \%)$, minimum $=0 \%$, and contour interval $=0.25(28.8 \%)$. The generalized two-dimensional Gaussian full model was fitted to the upwind and downwind side separately. For points on the $\mathrm{Y}$ axis $(\mathrm{X}=0)$, the two fitted values from each fitted model (upwind and downwind) were averaged together. Data for fitted map were transformed with the multiple infection correction $\ln [1 /(1-y)]$, in which $y=$ incidence of seed infection. Values in parentheses are back-transformed values. Each plot was $25 \times 25 \mathrm{~m}$, and the inoculated area was $1 \times 1 \mathrm{~m}$. Arrows represent north and average nightly wind vector during 1 to 31 July. 
generalized 2D Gaussian model, based on adjusted $r^{2}$ values. The submodel without the linear terms $\mathrm{X}$ and $\mathrm{Y}$ provided a similar fit. All models, except the classical Gaussian model, were significant $(P<0.05)$ (Table 1). However, when a $5 \times 5$ grid was used, the generalized 2D Gaussian model provided the best fit with a $r^{2}$ of 0.53 and was the best of all the models when compared with an adjusted $r^{2}$. Removal of either the quadratic or linear terms reduced the fit of the corresponding submodels. Both the stepwise regression procedures and the procedure maximizing the adjusted $r^{2}$ retained all six parameters of the full model. Both the modified inverse power and exponential model also provided a significant fit, but not the classical Gaussian model. When the grid size was reduced to $3 \times 3$, the modified inverse power model provided the best fit and was the only model that was significant $(P<0.05)$. The crosssection of this focus is shown in Figure 5.

Case 2: elliptical. The observed data and the data fitted using the generalized 2D Gaussian model are mapped in Figure 6A and B, respectively. The models are compared in Tables 3 and 4 . When using the $6 \times 6$ grid covering area outside the disease focus, only the modified inverse power model provided a significant fit, but the $r^{2}$ was very low $(0.16)$ (Table 3 ). However, when a $5 \times 5$ grid was used, the modified inverse power, exponential, and generalized 2D Gaussian model provided fairly equal fits to the observed data, $r^{2}=0.23$ to 0.25 . When the $\mathrm{X}$ and $\mathrm{Y}$ terms were removed from the generalized 2D Gaussian model, the fit of the model was increased, based on the adjusted $r^{2}$. Stepwise regression showed that just two parameters, $\mathrm{Y}^{2}$ and $F$, were sufficient for the submodel to provide a significant fit. When the grid size was reduced to $3 \times 3$, the generalized 2D Gaussian model clearly outperformed the other models, with a $r^{2}$ of 0.80 and an adjusted $r^{2}$ of 0.46 . Because of the reduced numbers of degrees of freedom, none of the other models were significant. At this scale, $\mathrm{X}, \mathrm{Y}$, and $\mathrm{Y}^{2}$ were retained by the procedure maximizing the adjusted $r^{2}$. Probabilities to enter

TABLE 5. Model performance with case 3, an anisotropic focus with one axis of symmetry, Ottawa, 1994

\begin{tabular}{lcccc}
\hline $\begin{array}{l}\text { Sample size/ } \\
\text { goodnes-of- } \\
\text { fit statistic }\end{array}$ & $\begin{array}{c}\text { Unmodified } \\
\text { inverse power }\end{array}$ & $\begin{array}{c}\text { Modified } \\
\text { inverse power }\end{array}$ & Exponential & $\begin{array}{c}\text { Classical } \\
\text { Gaussian }\end{array}$ \\
\hline$n=36$ & & & & \\
$\quad r^{2}$ & 0.11 & 0.30 & 0.20 & 0.15 \\
$\quad$ Adjusted $r^{2}$ & 0.09 & 0.27 & 0.17 & 0.12 \\
$P$ & 0.05 & $<0.01$ & 0.01 & 0.02 \\
$n=25$ & & & & \\
$r^{2}$ & 0.14 & 0.35 & 0.24 & 0.15 \\
$\quad$ Adjusted $r^{2}$ & 0.10 & 0.32 & 0.21 & 0.11 \\
$P$ & 0.08 & $<0.01$ & 0.01 & 0.06 \\
\hline
\end{tabular}

TABLE 6. Generalized two-dimensional Gaussian full model and submodels for case 3, an anisotropic focus with one axis of symmetry, Ottawa, 1994

\begin{tabular}{lccccc}
\hline Model/selection & $n$ & $\begin{array}{c}\text { Number of } \\
\text { parameters }^{\mathrm{a}}\end{array}$ & $r^{2}$ & Adjusted $r^{2}$ & $\begin{array}{c}P \text { value } \\
\text { for model }\end{array}$ \\
\hline Full & 34 & 12 & 0.50 & 0.24 & 0.08 \\
No X or Y & 34 & 8 & 0.37 & 0.20 & 0.07 \\
X and Y & 34 & 6 & 0.36 & 0.25 & 0.02 \\
Stepwise 0.15 & 34 & No model & & & \\
Stepwise 0.20 & 34 & 5 & 0.38 & 0.29 & 0.01 \\
Stepwise 0.25 & 34 & 5 & 0.38 & 0.39 & 0.01 \\
Max. adjusted $r^{2}$ & 34 & 6 & 0.44 & 0.34 & 0.01 \\
Full & 24 & 12 & 0.65 & 0.33 & 0.12 \\
No X or Y & 24 & 8 & 0.49 & 0.26 & 0.10 \\
X and Y & 24 & 6 & 0.39 & 0.22 & 0.09 \\
Stepwise 0.15 & 24 & No model & & & \\
Stepwise 0.20 & 24 & No model & & & \\
Stepwise 0.25 & 24 & 7 & 0.56 & 0.40 & 0.02 \\
Max. adjusted $r^{2}$ & 24 & 8 & 0.61 & 0.44 & 0.02 \\
\hline
\end{tabular}

${ }^{a}$ Includes intercept.

${ }^{b}$ For stepwise regressions, the number denotes the probability level at which regressors enter the model. and to stay of 0.25 were needed to retain the same submodel. The bilinear term XY, which indicates the orientation of the main axis of the focus in relation to the $(\mathrm{X}, \mathrm{Y})$ Cartesian system, was not retained by any submodel, showing the alignment of the orientation of the focus with the axes of sampling grid. The cross-sections of this focus are shown in Figure 7.

Case 3: anisotropic with one axis of symmetry. The observed data and the data fitted using the generalized 2D Gaussian model
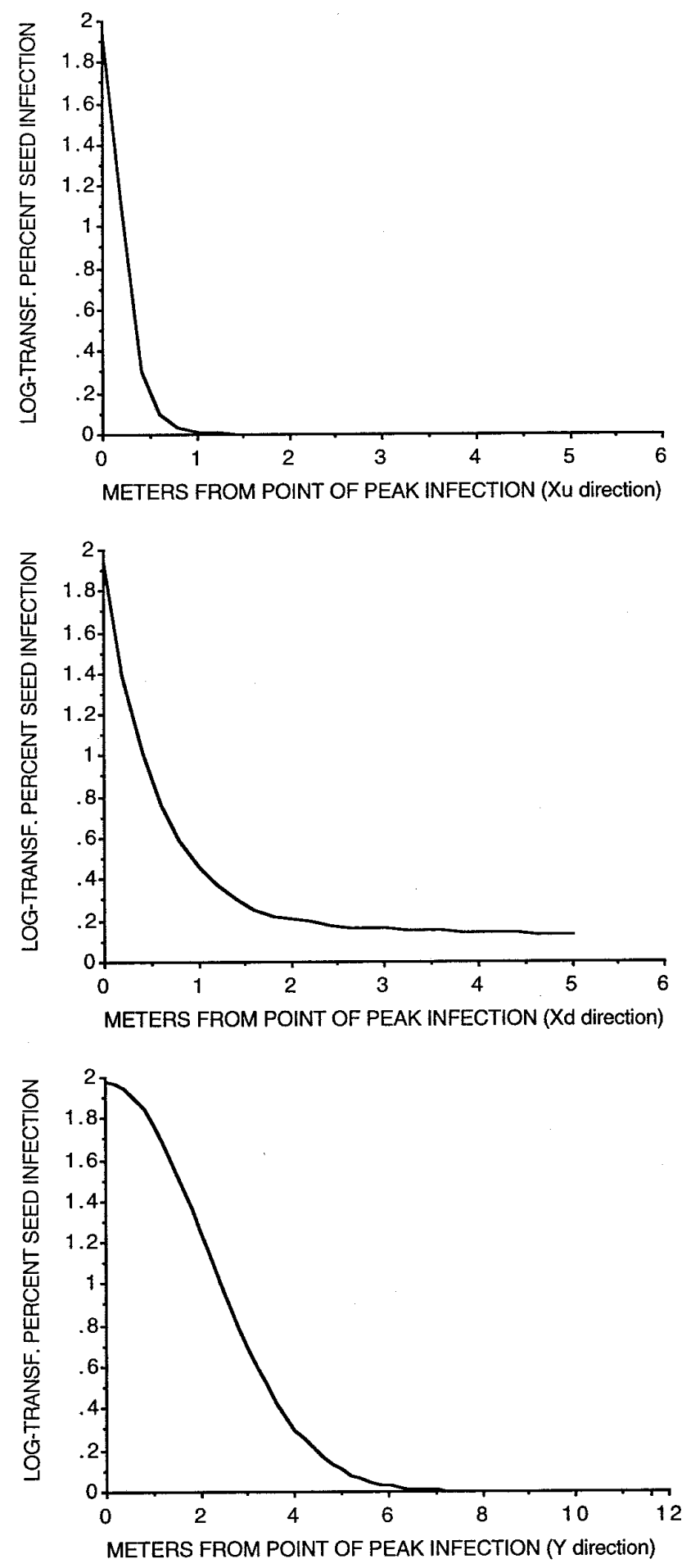

Fig. 9. One-dimensional gradients of spikelet infection derived from the generalized two-dimensional Gaussian full model fitted to the upwind and downwind side separately, for the anisotropic focus with one axis of symmetry in Figure 8. 
are mapped in Figure 8A and B, respectively. Models are compared in Tables 5 and 6 . With the $6 \times 6$ data grid, the full generalized 2D Gaussian model provided a fit similar to the modified inverse power model, although all models were significant. However, when the quadratic terms were removed, the adjusted $r^{2}$ increased slightly. The best fit was provided by a submodel with just three parameters besides the intercept: $\mathrm{Y}$ and $\mathrm{Y}^{2}$ from the downwind side and $X$ from the upwind side. When the grid size was reduced to $5 \times 5$, the generalized 2D Gaussian model also provided the best fit, with a $r^{2}$ of 0.65 . The cross-sections of this focus are shown in Figure 9.

Case 4: anisotropic with zero axis of symmetry. The observed data and the data fitted using the generalized 2D Gaussian model are mapped in Figure 10A and B, respectively. Models are com-
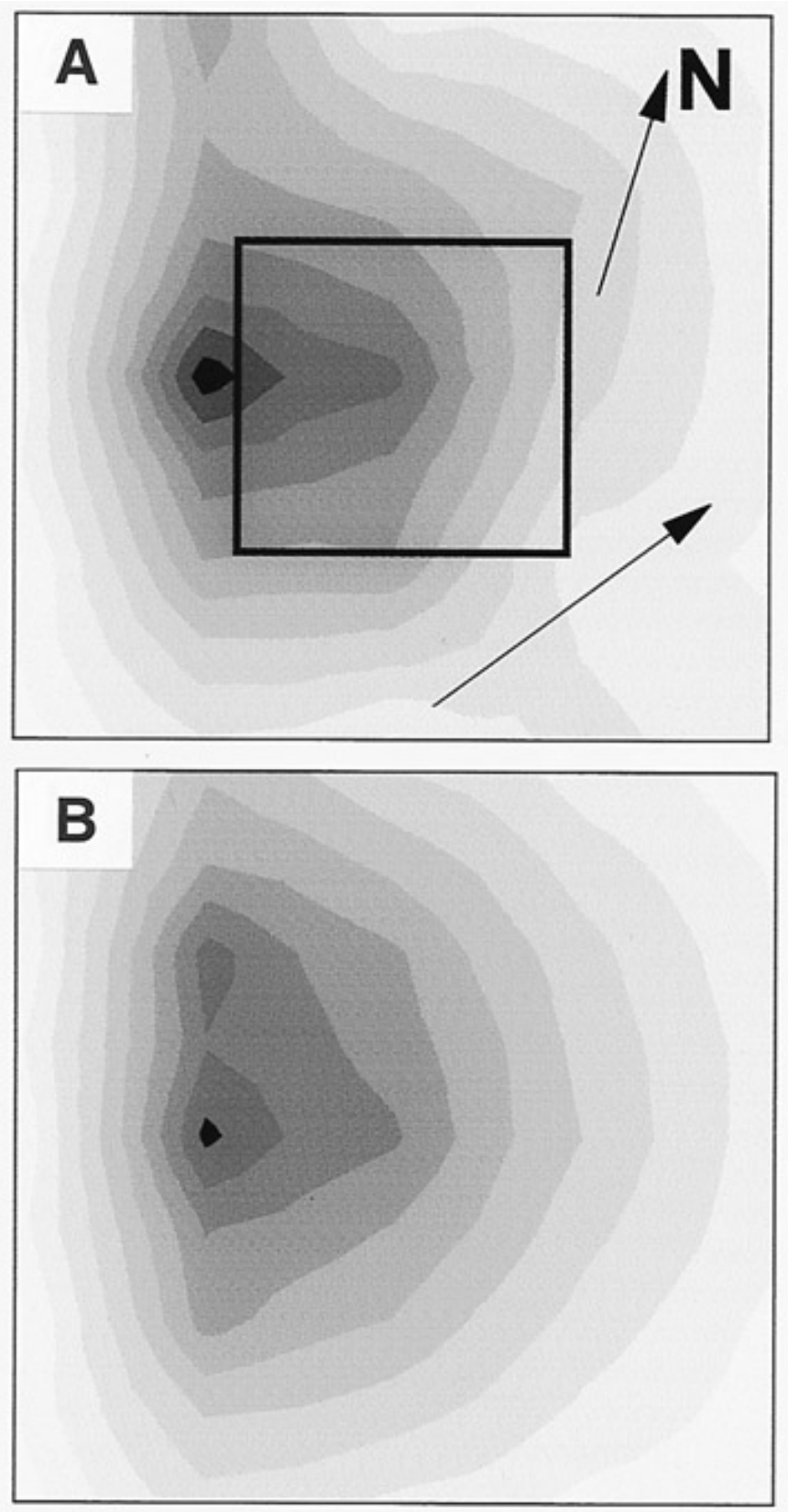

Fig. 10. Contour maps of incidence of spikelet infection derived from A, observed data and $\mathbf{B}$, model-fitted data of an anisotropic focus with zero axis of symmetry, Ste. Anne de Bellevue, 1993. A, Maximum isopath $=40.8 \%$, minimum $=1.2 \%$, and contour interval $=3.5 \%$. $\mathbf{B}$, Maximum isopath $=36 \%$, minimum $=1.1 \%$, and contour interval $=3.5 \%$. The generalized two-dimensional Gaussian full model was fitted to the upwind and downwind side separately. The plot was $10 \times 10 \mathrm{~m}$, and the inoculated area was $4 \times 4 \mathrm{~m}$. Arrows indicate north and the average nightly wind vector during 1 to 22 July. pared in Tables 7 and 8 . With the $5 \times 5$ grid, the generalized 2D Gaussian model provided by far the best fit, with a $r^{2}$ of 0.92 and an adjusted $r^{2}$ of 0.85 . Removal of the quadratic terms reduced the goodness-of-fit of the model. The best submodel was with five terms besides the intercept: $\mathrm{X}, \mathrm{Y}$, and $\mathrm{Y}^{2}$ on the upwind side and $\mathrm{X}$ and $\mathrm{Y}^{2}$ on the downwind side. The bilinear term $\mathrm{XY}$ was also retained by some submodels, although its contribution was not as high as the other parameters. The next best model, and the only other one that was significant, was the modified inverse power model, with a $r^{2}$ of 0.18 .

Spatial analysis of the residuals. No spatial autocorrelation in the residuals was detected for any of the best generalized 2D Gaussian model or submodel selected, according to Geary's $c$ coefficient at first distance class. However, there was significant autocorrelation in case 4 with the modified inverse power model $(n=25)$ and the classical Gaussian model $(n=9)$. Autocorrelation was also detected in case 3 with the exponential model $(n=9)$.

\section{DISCUSSION}

One of the limitations of most previous studies of disease gradients is that $1 \mathrm{D}$ measurements are taken spatially in one direction from the disease focus, or many directions are averaged together. Information about the 2D spread of disease has been inferred from these models. Lambert et al. (15) attempted to compensate for this by including compass direction in the observations, but this increased the coefficient of determination only slightly. Our approach was to use a grid sampling method to obtain data in two dimensions and to develop a versatile model that would adjust for differences in the gradient, depending on the direction from the focus.

In the simplest scenario, the gradients of the disease focus would be circular in cross-sections (Figs. 3 and 4), with the gradient being the same in all directions away from the peak of the focus. This was only observed in 1 out of 12 experimental plots with ascospore inoculum at two locations over 3 years. This focal shape may not be common with airborne spores such as ascospores, since the wind will influence the dispersal of inoculum and create an asymmetry. However, this type of focus may be more common with splash-dispersed spores. In the case of the focus with the circular cross-section, the performance of the generalized 2D Gaussian model was maximum when the data was scaled down to 25 data points, to eliminate some of the data points outside of the focus. In

TABLE 7. Model performance with case 4, an anisotropic focus with zero axis of symmetry, Ste. Anne de Bellevue, 1993

\begin{tabular}{lcccc}
\hline $\begin{array}{l}\text { Sample size/ } \\
\text { goodnes-of- } \\
\text { fit statistic }\end{array}$ & $\begin{array}{c}\text { Unmodified } \\
\text { inverse power }\end{array}$ & $\begin{array}{c}\text { Modified } \\
\text { inverse power }\end{array}$ & Exponential & $\begin{array}{c}\text { Classical } \\
\text { Gaussian }\end{array}$ \\
\hline$n=25$ & & & & \\
$r^{2}$ & 0.08 & 0.18 & 0.13 & 0.08 \\
Adjusted $r^{2}$ & 0.04 & 0.14 & 0.09 & 0.04 \\
$P$ & 0.17 & 0.04 & 0.08 & 0.17 \\
\hline
\end{tabular}

TABLE 8. Generalized two-dimensional Gaussian full model and submodels for case 4, an anisotropic focus with zero axis of symmetry, Ste. Anne de Bellevue, 1993

\begin{tabular}{lccccc}
\hline Model/selection & $n$ & $\begin{array}{c}\text { Number of } \\
\text { parameters }^{\mathrm{a}}\end{array}$ & $r^{2}$ & Adjusted $r^{2}$ & $\begin{array}{c}P \text { value } \\
\text { for model }\end{array}$ \\
\hline Full & 25 & 12 & 0.92 & 0.85 & $<0.001$ \\
No X or Y & 25 & 8 & 0.91 & 0.87 & $<0.001$ \\
X and Y & 25 & 6 & 0.79 & 0.74 & $<0.001$ \\
Stepwise 0.15 & 25 & 7 & 0.89 & 0.86 & $<0.001$ \\
Stepwise 0.20 & 25 & 7 & 0.89 & 0.86 & $<0.001$ \\
Stepwise 0.25 & 25 & 8 & 0.89 & 0.84 & $<0.001$ \\
Max. adjusted $r^{2}$ & 25 & 10 & 0.91 & 0.86 & $<0.001$ \\
\hline
\end{tabular}

a Includes intercept.

${ }^{\mathrm{b}}$ For stepwise regressions, the number denotes the probability level at which regressors enter the model. 
this experiment, the disease focus only occupied about $30 \mathrm{~m}^{2}$, compared with the total area of $625 \mathrm{~m}^{2}$. But when the entire plot is included, with large areas of no or low disease, the modified inverse power model tends to perform the same or slightly better than the generalized 2D Gaussian model.

In the second case, a focus with an elliptical (anisotropic with two axes of symmetry) shape was only seen when macroconidial inoculum was directly applied to the heads. Since there is little or no secondary spread and the inoculum is not affected by wind vectors, unlike ascospores, a tight symmetrical focus was formed. The full generalized 2D Gaussian model more closely fitted the observed data when the covered area was scaled down to include only nine data points, which included just the disease focus. But the submodel reduced to only two terms, $\mathrm{Y}^{2}$ and $F$, outperformed the full model, indicating that the gradients away from the center, at least in one direction, showed a Gaussian shape. Otherwise, the modified inverse power model provided a better fit. The real strength of the generalized 2D Gaussian model was seen with anisotropic foci with one or zero axis of symmetry, the type most commonly seen in our work with ascospore-inoculated plots, which are influenced by wind direction. In such cases, our model clearly provided a better fit of the data than the modified inverse power model. For example, in the data set from Ste. Anne de Bellevue (case 4) (figure 1B in literature citation 6), our model had an adjusted $r^{2}$ value of 0.85 , compared with a value of 0.14 with the modified inverse power model. In the case of Ottawa (case 3), scaling down the data to 25 points slightly increased the fit of the latter model. This demonstrates the importance of data selection and scaling on model performance. Clearly, the generalized 2D Gaussian model is more versatile and adaptable to anisotropic disease foci with reduced symmetry or no symmetry at all.

Compared with the generalized 2D Gaussian model and the modified inverse power model, the other models generally provided lower goodness-of-fit. The unmodified inverse power model greatly overestimates the disease at the source and may not be suitable for steep gradients such as the ones described here (16). Mundt (18) corrected for this with a truncation factor $c$ to provide for a finite intercept at $x^{\prime}=0$ in equation 2 . He found that this modified model provided a better fit of primary disease gradients of wheat leaf rust than the exponential model. Fitt et al. (8) also compared the unmodified inverse power model with the exponential model for gradients of 325 data sets. They concluded that neither model provided a better fit overall, although gradients with splash-dispersed spores were better described with the exponential model. Unlike Fitt et al. (8), we found the exponential model to be superior to the unmodified inverse power model in every case, possibly because our measurements of disease were relatively close to the source and the gradients were fairly steep. The classical Gaussian model of Kampmeijer and Zadoks (13) generally provided a poorer fit than the modified inverse power or exponential models, even with symmetrical foci. In some cases, this may come from the sharpness of the peak, which results in the predominance of linear parameters over the quadratic parameters as in the exponential function. The generalized 2D Gaussian model does not suffer from this drawback of the classical 1D Gaussian model because of the presence of both types of parameters plus a bilinear one in equation 6 .

What is the biological significance of the different model parameters? The shape of the disease gradients determines the presence or absence of specific parameters in the model. These parameters can be eliminated from the full model, based on stepwise regression or by testing the fit of various submodels lacking these parameters. For example, if only the $\mathrm{X}$ and $\mathrm{Y}$ linear terms are retained with no quadratic terms, this reflects an exponential gradient that has a sharp peak in disease very close to the center of the focus and high levels of disease limited to a very small area. The decrease in disease is then very drastic, moving a very short distance away from the peak focus. On the other hand, retaining the quadratic terms $\mathrm{X}^{2}$ and $\mathrm{Y}^{2}$ and the bilinear term $\mathrm{XY}$ without the linear terms reflects a Gaussian gradient, in which there is a flat peak of high disease levels at a limited distance from the focus center. The distance at which the disease declines to a given level may also be longer with a Gaussian gradient. The bilinear term XY can provide information about the orientation of the main axis of the focus in relation to the axes of the sampling grid, and the corresponding angle (Fig. 2B) can be described according to the equation

$$
\varphi=1 / 2 \tan ^{-1}[C /(A-B)]
$$

in which the estimates of parameters $A, B$, and $C$ come from the fitting of equation 10 .

For our data sets, stepwise regression was able to remove some unneeded parameters from the model for these foci, but in most cases, a mixture of linear and quadratic terms was retained. In the anisotropic foci with one and zero axis of symmetry (cases 3 and 4), the term $\mathrm{X}$ was retained for the model on the upwind side, but not $X^{2}$. This indicates that the gradient away from the focus in the upwind direction could be described by an exponential function. However, in the model of the downwind side, the $\mathrm{Y}^{2}$ term was retained in both cases, along with either $\mathrm{Y}$ or $\mathrm{X}$. This indicates that the gradient in the direction approximately perpendicular to the wind can be described by a Gaussian function, whereas an exponential function is retained in the direction parallel to the wind.

The bilinear term XY was retained by some submodels for the Ste. Anne de Bellevue plot (case 4). This indicates that the orientation of the main axis of the focus, which was correlated with the wind direction, did not align with the axes of the sampling grid. For the example in Figure 10, $A=0.0015, B=0.027$, and $C=-0.025$, so $\varphi=22.5^{\circ}$. The main axis of this focus was slightly shifted to the NE-SW, compared with the horizontal axis X of the sampling grid.

In conclusion, the generalized 2D Gaussian model is an improvement over existing models for spatially describing disease foci of G. zeae in wheat. Unlike the existing models, it contains a larger number of parameters (quadratic, bilinear, and linear) and so can be adjusted to account for the typical anisotropy of foci arising from wind-dispersed spores. This model may be useful for describing and predicting the dispersal of inoculum of G. zeae for short distances. We hope that this model stimulates further work with 2D spatial gradients, with the hope of better understanding 2D spread of plant diseases.

\section{ACKNOWLEDGMENTS}

This work was supported by a grant from the Natural Sciences and Engineering Research Council of Canada to T. C. Paulitz and P. Dutilleul and a grant to P. Dutilleul from the Fonds pour la formation de chercheurs et l'aide à la recherche du Québec. We thank H. Cohen-Rimmer for her assistance with the graphics and C. Mundt for helpful suggestions. We also thank the three anonymous reviewers whose comments helped improve the development of some specific aspects of our model and its presentation.

\section{LITERATURE CITED}

1. Alderman, S. C., Nutter, Jr., F. W., and Labrinos, J. L. 1989. Spatial and temporal analysis of spread of late leaf spot of peanut. Phytopathology 79:837-844.

2. Cliff, A. D., and Ord, J. K. 1981. Spatial Processes. Models and Applications. Pion Limited, London.

3. Draper, N., and Smith, H. 1981. Applied Regression Analysis. 2nd ed. John Wiley \& Sons, New York.

4. Durbin, J., and Watson, G. S. 1951. Testing for serial correlation in least squares regression. Biometrika 37:409-428.

5. Dutilleul, P., and Legendre, P. 1993. Spatial heterogeneity against heteroscedasticity. An ecological paradigm versus a statistical concept. Oikos 66:152-171.

6. Fernando, W. G. D., Paulitz, T. C., Seaman, W. L., Dutilleul, P., and Miller, J. D. 1997. Head blight gradients caused by Gibberella zeae from area sources of inoculum in wheat field plots. Phytopathology 87:414-421.

7. Ferrandino, F. J. 1993. Dispersive epidemic waves: I. Focus expansion within a linear planting. Phytopathology 83:795-802.

8. Fitt, B. D. L., Gregory, P. H., Todd, A. D., McCartney, H. A., and 
Macdonald, O. C. 1987. Spore dispersal and plant disease gradients: A comparison between two empirical models. J. Phytopathol. 118:227-242.

9. Geary, R. C. 1954. The contiguity ratio and statistical mapping. The Incorporated Statistician 5:115-145.

10. Gottwald, T. R. 1995. Spatio-temporal analysis and isopath dynamics of citrus scab in nursery plots. Phytopathology 85:1082-1092.

11. Gregory, P. H. 1948. The multiple-infection transformation. Ann. Appl. Biol. 35:412-417.

12. Gregory, P. H. 1968. Interpreting plant disease dispersal gradients. Annu. Rev. Phytopathol. 6:189-212.

13. Kampmeijer, P., and Zadoks, J. C. 1977. EPIMUL, a simulator of foci and epidemics in mixtures of resistant and susceptible plants, mosaics and multilines. Centre Agric. Publ. and Documentation, Wageningen, the Netherlands.

14. Kiyosawa, S., and Shiyomi, M. 1972. A theoretical evaluation of the effect of mixing a resistant variety with a susceptible variety for controlling plant diseases. Ann. Phytopathol. Soc. Jpn. 38:41-51.

15. Lambert, D. H., Villareal, R. L., and Mackenzie, D. R. 1980. A general model for gradient analysis. J. Phytopathol. 98:150-154.

16. McCartney, H. A., and Fitt, B. D. L. 1985. Construction of dispersal models. Adv. Plant Pathol. 3:107-143.

17. Minogue, K. P. 1986. Disease gradients and the spread of disease. Pages 285-310 in: Plant Disease Epidemiology, Vol. 1: Population Dynamics and Management. K. J. Leonard and W. E. Fry, eds. Macmillan Publishing Company, New York.

18. Mundt, C. C. 1989. Use of the modified Gregory model to describe primary disease gradients of wheat leaf rust produced from area sources of inoculum. Phytopathology 79:241-246.

19. Sutton, J. D. 1982. Epidemiology of wheat head blight and maize ear rot caused by Fusarium graminearum. Can. J. Plant Pathol. 4:195-209. 